Open Access

\title{
$\beta$-Catenin is required for the differentiation of iNKT2 and iNKT17 cells that augment IL-25-dependent lung inflammation
}

Rosa Berga-Bolaños ${ }^{1}$, Archna Sharma ${ }^{1,7}$, Farrah C. Steinke ${ }^{2}$, Kalyani Pyaram³ ${ }^{3}$ Yeung-Hyen Kim³, Dil A. Sultana ${ }^{1,8}$, Jessie X. Fang ${ }^{4}$, Cheong-Hee Chang ${ }^{3}$, Hai-Hui Xue ${ }^{2}$, Nicola M. Heller ${ }^{4}$ and Jyoti Misra Sen ${ }^{1,5,6^{*}}$

\begin{abstract}
Background: Invariant Natural Killer T (iNKT) cells have been implicated in lung inflammation in humans and also shown to be a key cell type in inducing allergic lung inflammation in mouse models. iNKT cells differentiate and acquire functional characteristics during development in the thymus. However, the correlation between development of iNKT cells in the thymus and role in lung inflammation remains unknown. In addition, transcriptional control of differentiation of iNKT cells into iNKT cell effector subsets in the thymus during development is also unclear. In this report we show that $\beta$-catenin dependent mechanisms direct differentiation of iNKT2 and iNKT17 subsets but not iNKT1 cells.
\end{abstract}

Methods: To study the role for $\beta$-catenin in lung inflammation we utilize mice with conditional deletion and enforced expression of $\beta$-catenin in a well-established mouse model for IL-25-dependen lung inflammation.

Results: Specifically, we demonstrate that conditional deletion of $\beta$-catenin permitted development of mature iNKT1 cells while impeding maturation of iNKT2 and 17 cells. A role for $\beta$-catenin expression in promoting iNKT2 and iNKT17 subsets was confirmed when we noted that enforced transgenic expression of $\beta$-catenin in iNKT cell precursors enhanced the frequency and number of iNKT2 and iNKT17 cells at the cost of iNKT1 cells. This effect of expression of $\beta$-catenin in iNKT cell precursors was cell autonomous. Furthermore, iNKT2 cells acquired greater capability to produce type-2 cytokines when $\beta$-catenin expression was enhanced.

Discussion: This report shows that $\beta$-catenin deficiency resulted in a profound decrease in iNKT2 and iNKT17 subsets of iNKT cells whereas iNKT1 cells developed normally. By contrast, enforced expression of $\beta$-catenin promoted the development of iNKT2 and iNKT17 cells. It was important to note that the majority of iNKT cells in the thymus of C57BL/6 mice were iNKT1 cells and enforced expression of $\beta$-catenin altered the pattern to iNKT2 and iNKT17 cells suggesting that $\beta$-catenin may be a major factor in the distinct pathways that critically direct differentiation of iNKT effector subsets.

Conclusions: Thus, we demonstrate that $\beta$-catenin expression in iNKT cell precursors promotes differentiation toward iNKT2 and iNKT17 effector subsets and supports enhanced capacity to produce type 2 and 17 cytokines which in turn augment lung inflammation in mice.

Keywords: Beta-catenin, iNKT cells, iNKT2, iNKT17, IL-17RB

\footnotetext{
* Correspondence: Jyoti-Sen@nih.gov

'Immune Cells and Inflammation Section, National Institute on Aging,

National Institutes of Health, Baltimore, MD 21224, USA

${ }^{5}$ Department of Medicine, The Johns Hopkins University School of Medicine

Baltimore, MD 21287, USA

Full list of author information is available at the end of the article
} 


\section{Background}

Invariant Natural killer $\mathrm{T}$ (iNKT) cells participate in both innate and adaptive immune functions [1-4]. iNKT cells develop from $\mathrm{CD} 4^{+} \mathrm{CD} 8^{+}$double positive (DP) thymocytes that express an invariant T-cell receptor (TCR). In mice $T C R \alpha$ chain $V \alpha 14-J \alpha 18$ pairs with $\mathrm{V} \beta 8, \mathrm{~V} \beta 7$ or $\mathrm{V} \beta 2$ to generate a TCR repertoire that recognizes glycolipid antigens presented by the MHC class I-like molecule CD1d [5-7]. Recognition of lipid antigens presented by CD1d expressed on DP thymocytes leads to commitment to the iNKT lineage. Immature iNKT0 cells then receive developmental cues that control differentiation into type1 iNKT1, type2 iNKT2 and type17 iNKT17 cells that produce cytokines that are similar to those produced by mature effector CD4 T cells after encounter with antigen [8-10]. However, in contrast to effector CD4 T cells, iNKT cells gain the ability to express cytokines during development and respond rapidly when stimulated [1, 11]. Whereas the specific transcription factors that regulate cytokine production by each subset of iNKT cells are well defined, the transcriptional programs that control iNKT cell generation and differentiation into effector cells remain to be delineated.

The connection between iNKT cells and the development of asthma or allergic lung inflammation in mice was revealed and non-adaptive immune cellular candidates were sought to understand how non-allergic environmental triggers could lead to asthma. Typical allergen sensitization and challenge models could not explain why air pollution, viruses and obesity enhanced the disease. These studies uncovered roles for iNKT and innate lymphoid cell populations in the lung in modulating allergic lung inflammation. Since then, numerous human studies have attempted to correlate frequency, function and location of iNKT cells to asthma severity. Akbari et al. showed that lung CD4+ iNKT cells from asthmatics secreted both IL-4 and IL-13 but little IFN- $\gamma$ on $\alpha \mathrm{GalCer}$ stimulation [12]. However, conclusions about the contribution of peripheral blood iNKT cells from asthmatics to the severity of asthma have been equivocal [13-16]. Nonetheless, iNKT cells play an essential role in viral and pediatric exacerbations of asthma and in severe asthma [17]. Defining their contribution to allergic lung inflammation is of critical clinical importance to finding novel approaches to regulate the number and function of iNKT cells. Furthermore, understanding the molecular mechanisms that regulate how iNKT cells develop and differentiate is key to controlling the number and frequency of these cells.

$\beta$-Catenin is ubiquitously expressed and in T cells is augmented in response to TCR signals and regulates $\mathrm{T}$ cell development in the thymus [18-23]. In addition to conventional $\mathrm{T}$ cells, the thymus is also the site for innate immune cell development [24, 25]. We have demonstrated that $\beta$-catenin expression regulates the generation of innate-like CD8 (iCD8) thymocytes [26]. However, a role for $\beta$-catenin in the generation and differentiation of iNKT cells in the thymus remains to be defined.

In this study we report that mice with enforced expression of $\beta$-catenin in iNKT cell precursors ( $\beta$-CAT-Tg) experience augmented IL-25-dependent lung inflammation. iNKT cells in the lung produced increased amounts of IL-13 and IL-17 and presented intensified inflammation at alveolar sites and surrounding blood vessels. We demonstrate that augmented lung inflammation correlated with enhanced generation of iNKT2 and iNKT17 cells in $\beta$-CAT-Tg thymus at the expense of iNKT1 cells. Using mice in which $\beta$-catenin was deleted in iNKT cell precursors $(\beta-\mathrm{CAT}-\mathrm{KO})$ we show that expression of $\beta$-catenin is selectively required for differentiation of iNKT2 and iNKT17 cells as iNKT1 cells developed normally. In conclusion, this study demonstrates that $\beta$-catenin expression was required to promote differentiation and functional maturation of iNKT2 and iNKT17 cells that contributed to augmented IL-25-dependent lung inflammation.

\section{Methods}

Mice

$\beta$-CAT-Tg mice, expressing an activated form of $\beta$ catenin in thymocytes and T cells from the proximal $L c k$ promoter, have been previously described [27]. $\beta$-CATcKO mice were generated by breeding mice bearing a LoxP-flanked gene encoding $\beta$-catenin $\left(\beta-\mathrm{CAT}^{\text {flox/flox }}\right.$ ) [28] with mice expressing the Cre recombinase under the control of the $C d 4$ promoter (CD4-Cre mice). All the mice used are on a C57BL/6 genetic background. CD45.1+ C57BL/6.SJL mice were purchased from Taconic. $\mathrm{CD} 45.1+2+$ mice were generated by breeding C57BL/6.SJL mice with C57BL/6 mice. Age-matched (710 weeks old) littermate controls or C57BL/6 mice were used in all experiments. All mice were bred and maintained in animal facility at the National Institute on Aging (NIA). The studies were carried out in accordance with the recommendations in the Guide for the Care and Use of Laboratory Animals (NRC 2010). The protocol was approved by the Animal Care and Use Committee of the NIA Intramural Research Program, NIH. This program is fully accredited by the Association for Assessment and Accreditation of Laboratory Animal Care International (File 000401), registered by the United States Department of Agriculture (51-F-0016) and maintains an assurance with the Public Health Service (A4149-01).

\section{Flow cytometry}

Single-cell suspensions were prepared from thymus and spleens as per standard protocols. Hepatic lymphocytes 
were isolated from livers that were homogenized, filtered through nylon mesh and washed in PBS with $1 \%$ FBS. Cells were then resuspended in $44 \%$ Percoll (GE Healthcare Bio-Sciences AB, Uppsala, Sweden), underlaid with $66 \%$ Percoll, and centrifuged for $20 \mathrm{~min}$ at $2000 \mathrm{rpm}$. Cells at the interface were collected and washed. Cells were stained, acquired on a FACSCantoII (Becton Dickinson) and analyzed with FlowJo (Treestar). Dead cells were excluded using the Fixable Viability Dye eFluor ${ }^{\circ} 506$ (eBioscience). The following antibodies and their isotype controls conjugated to FITC, PE, PerCP-Cy5.5, PE-Cy7, APC, APC-Cy7 or Pacific Blue (from BD Biosciences, eBioscience or BioLengend) were used for staining: antiCD4 (GK1.5), anti-CD8 $\alpha$ (53-6.7), anti-TCR $\beta$ (H57597), anti-CD1d (1B1), anti-Siglec-F (E50-2440), antiLy6G (1A8), anti-CD11c (N418), anti-CD11b (M1/70), anti-CD19 (6D5), anti-IFN- $\gamma$ (XMG1.2), anti-IL-4 (11B11), anti-IL-13 (eBio13A) and anti-IL-17A (TC1118H10.1). Anti-IL-17RB-APC (752101) and its isotype control were purchased from R\&D Systems. PE- or APC- conjugated mouse CD1d tetramers loaded with glycolipid PBS-57 (CD1d-tet) were obtained from the tetramer facility of the US National Institutes of Health. In brief, cells were incubated with FC block and stained with antibodies, and then fixed with $2 \%$ paraformaldehyde. For IFN- $\gamma$, IL-4, IL-13 and IL-17A intracellular staining, cells were permeabilized, stained and fixed using the BD Cytofix/Cytoperm kit (BD Biosciences). For PLZF and T-bet intracellular staining, cells were permeabilized and stained with anti-PLZF (D-9) (Santa Cruz Biotechnology, Inc.) plus FITC anti-mouse (BD Biosciences) and APC-conjugated anti-T-bet (eBio4B10) purchased from eBioscience, using the Foxp3 Staining Buffer kit (eBioscience).

\section{Bone marrow chimeras}

For the BM chimera experiments, each recipient mouse received $2 \times 10^{6}$ whole bone marrow cells from a single donor in $400 \mu \mathrm{l}$ of PBS through i.v. injection. For the BM mixed chimera experiments, BM cells from two different types of donor mice were mixed at 1:1 ratio. Each recipient mouse received $9 \times 10^{6}$ cells in $250 \mu \mathrm{l}$ of PBS through i.v. injection. In all experiments, CD45 congenic markers were used to distinguish cells derived from the different sources. All BM chimeras were reconstituted for at least 7 weeks before analysis.

\section{In vitro PMA- and ionomycin-induced activation assay}

For in vitro stimulation, thymocytes were cultured in $\mathrm{T}$ cell medium (RPMI 1640 with $10 \%$ FBS, HEPES, penicillin and streptomycin, L-glutamine and 2-mer captoethanol) and stimulated for $5 \mathrm{~h}$ with phorbol 12 myristate 13 -acetate (PMA) $(50 \mathrm{ng} / \mathrm{ml})$ and ionomycin
$(1 \mu \mathrm{M})$. For intracellular cytokine staining, Brefeldin A was added for the final $3.5 \mathrm{~h}$.

\section{In vivo a-Galactosylceramide stimulation}

Three micrograms of $\alpha$-Galactosylceramide in $200 \mu \mathrm{l}$ PBS were intraperitoneally injected into mice. Mice were sacrificed to obtain spleen $3 \mathrm{~h}$ after injection and splenocytes were stained for CD1d-tet, TCR $\beta, \mathrm{CD} 4, \mathrm{CD} 8 \alpha$, IL$17 R B$ and IL-4.

\section{AHR induction}

The OVA/IL-25-induced AHR model was performed as previously described [29] with modification. Mice were immunized with $50 \mu \mathrm{g} / 2 \mathrm{mg}$ i.p. OVA/alum twice (days 0 and 7), then treated with PBS or $2 \mu \mathrm{g}$ IL- 25 via i.p. at day 14 and finally challenged twice with $50 \mu \mathrm{g}$ intranasal OVA (days 16 and 17). At day 18, analysis was performed. The mouse trachea was cannulated and lungs were lavaged with $1.5 \mathrm{ml}$ cold $\mathrm{PBS}$ to obtain the BAL fluid from which, after centrifugation, cells were stained for flow cytometry with anti-TCR $\beta$, anti-CD19, antiLy6G, anti-CD11b, anti-CD11c and anti-Siglec-F. The left lung lobe was inflated with $1 \%$ low-melt agarose and fixed in formalin until embedded in paraffin to do sections that were stained with Hematoxylin and Eosin and Periodic acid Schiff. Photographs were taken with a Zeiss Axiophot microscope (lens: 10x Zeiss Plan Apo N.A. 0.32; lens: $25 x$ oil Zeiss Plan Apo N.A. .80) with a Prog Res C14 digital camera attached to the scope. Camera software was Adobe Photoshop 6.0. Lymphocytes from the other lung lobes were isolated by digestion with RPMI medium containing collagenase II and DNase I followed by a lympholyte separation prior to staining for flow cytometry analysis.

\section{Statistics}

Statistical significance was determined by the Student's $t$-test.

\section{Results \\ $\beta$-CAT-Tg mice show an exacerbated response to IL-25-dependent AHR induction}

iNKT cells that migrate to the lung tissue are known to cause IL-25-dependent airway hyperresponsiveness (AHR). The receptor for IL-25 is IL-17RB, which is present in certain iNKT cell subsets [29]. IL-25 is both necessary and sufficient for Th2 cytokine production, eosinophilic airway inflammation and AHR [30-32]. To determine if enforced expression of $\beta$-catenin affects iNKT cell function we analyzed transgenic mice that express a proximal $L c k$-driven stabilized mutant form of $\beta$-catenin in thymocytes and T cells ( $\beta$-CAT-Tg). We sensitized mice twice with OVA, along with treatment with IL-25 and finally challenged the mice 
intranasally with OVA. At the end of the protocol, we evaluated several hallmark characteristics of allergic lung inflammation. Histological analysis of lung sections from sensitized and challenged control and $\beta$ CAT-Tg mice showed typical features of allergic lung inflammation, which were particularly exacerbated in the $\beta$-CAT-Tg animals that were treated with IL-25 (Fig. 1a, left). Hematoxylin and eosin and Periodic acid Schiff (PAS) staining of the lung tissue revealed greater infiltration of immune cells and a significant increase in the number of mucus-producing cells in the lungs of IL-25 treated $\beta$-CAT-Tg mice compared to control mice (Fig. 1a, right). We quantified the immune cells infiltrating the airways by counting cells in the bronchoalveolar lavage fluid (BALF). We noticed an increase in all of these infiltrating cell types in the BALF from the challenged $\beta$ CAT-Tg mice (Fig. 1b, top). We wished to analyze the effect of the loss-of-function of $\beta$-catenin in AHR. To this end, we generated $\beta-\mathrm{CAT}^{\text {flox/flox }} \mathrm{CD} 4-\mathrm{Cre}^{+}$mice (referred to as $\beta$-catenin conditional knock-out, $\beta$-CAT-cKO). When the same experiment was performed in $\beta$-CAT-cKO mice, we did not see an increase in infiltration of cells compared to treated controls (Fig. 1b, bottom). These data suggest that $\beta$-CAT-Tg mice experience greater inflammation when challenged with OVA and IL-25 compared to control.

We further analyzed lung cell suspensions to detect the cytokine production by iNKT cells in the tissue. IFN$\gamma$ and IL-4 production was comparable in control, $\beta$ CAT-Tg and $\beta$-CAT-cKO mice challenged with OVA and IL-25 (Fig. 1c and data not shown). Strikingly, IL-
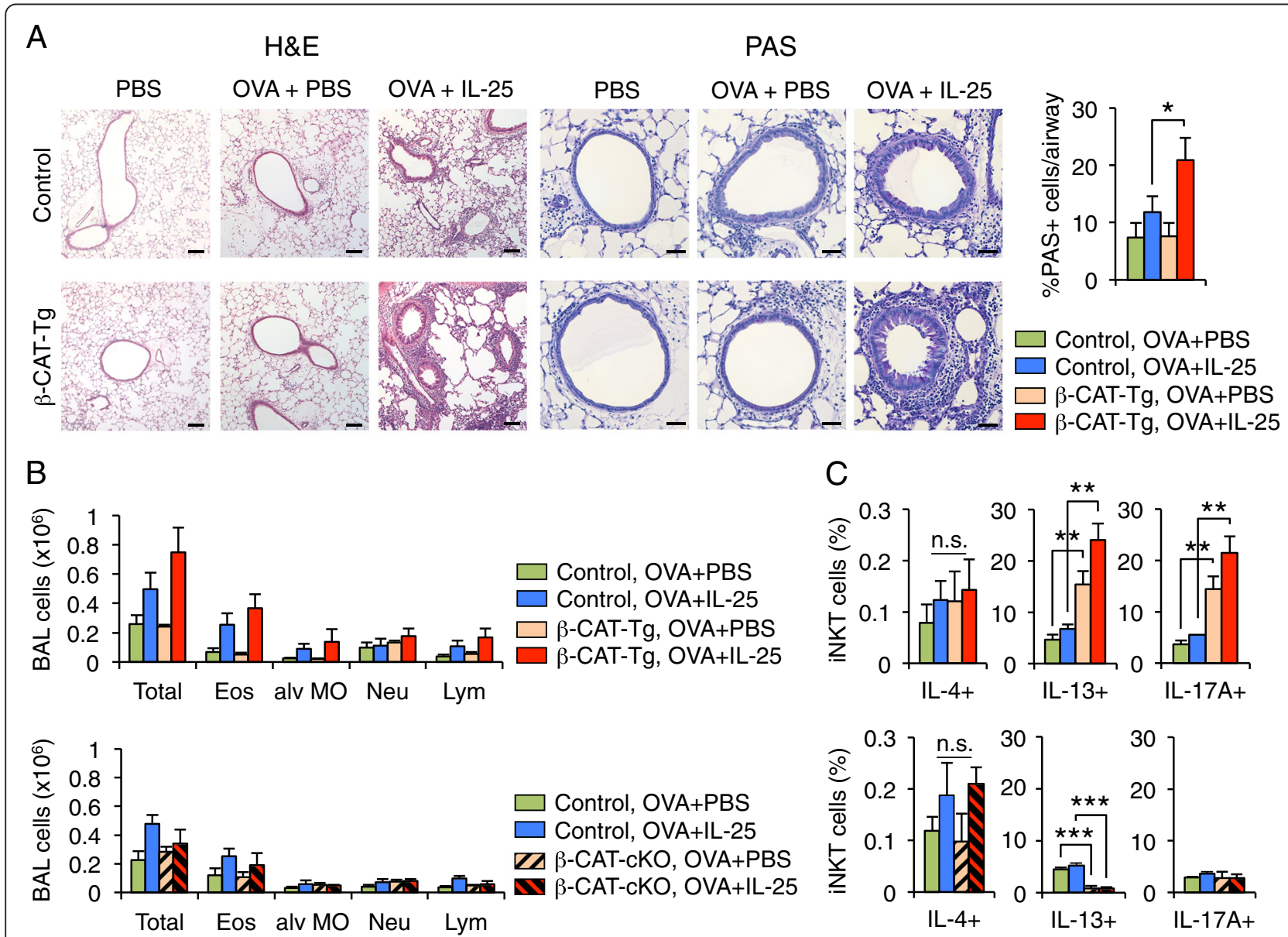

Fig. $1 \beta$-CAT-Tg mice show an exacerbated response to IL-25-dependent AHR induction. $\beta$-CAT-Tg, $\beta$-CAT-cKO and control mice were sensitized with OVA, treated with IL-25 or PBS and finally challenged with OVA to induce airway hyperreactivity (AHR). a Histological analysis of lung tissues with hematoxylin and eosin (H\&E) and periodic acid Schiff (PAS) staining. Representative control and $\beta$-CAT-Tg sections are shown. H\&E bars, $100 \mu \mathrm{m}$; PAS bars, $100 \mu \mathrm{m}$. Graph shows percentage of PAS-positive cells per total number of cells in the epithelium of the airway, counting 5-7 airways per lung section (mean \pm sem). b Total cellularity (Total) and cell numbers of eosinophils (Eos), alveolar macrophages (alv MO), neutrophils (Neu) and lypmhocytes (Lym) present in bronchoalveolar lavage (BAL) fluid ( $n=5$, mean \pm sem) from control and $\beta$-CAT-Tg (top) and control and

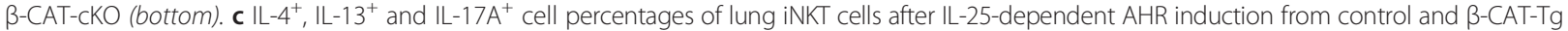
(top) and control and $\beta$-CAT-cKO (bottom) $(n=5$, mean \pm sem). AHR experiment was performed twice, with $3-5$ mice per group in each independent experiment. ${ }^{*} P<.05 ;{ }^{*} P<.01$ 
13-expressing iNKT cells that have been specially implicated in IL-25-dependent AHR induction [29, 33, 34] were significantly enhanced in iNKT cells from lung cell suspensions from $\beta$-CAT-Tg mice (Fig. 1c, top). By contrast IL-13-producing iNKT cells in $\beta$-CAT-cKO mice were fewer and unresponsive (Fig. 1c, bottom). Finally, IL-17A was also largely produced by $\beta$-CAT-Tg iNKT cells (Fig. 1c). Thus, $\beta$-CAT-Tg IL-17RB ${ }^{+}$iNKT cells contribute to a more robust allergic inflammatory response in the lungs of allergen-challenged $\beta$-CAT-Tg mice compared to control mice. The allergic response was particularly aggravated in CAT-Tg mice in the presence of IL-25 (Fig. 1a and c). These data demonstrate that in mice with enforced expression of $\beta$-catenin functional iNKT cells lead to a more robust response to IL-25dependent AHR challenge, with all measured parameters of allergic inflammation enhanced, including higher iNKT production of Th2 and Th17 cytokines.

\section{$\beta$-Catenin expression regulates the differentiation of effector iNKT2 and iNKT17 cells}

To determine the specific role of $\beta$-catenin in iNKT cell development, we studied thymic iNKT cells in mice with
T-cell conditional deletion or enforced expression of $\beta$-catenin. $\beta$-CAT-cKO mice showed similar proportions and total cell numbers of thymocytes (Fig. 2a, numbers on top of dot plots) and mature $\mathrm{T}$ cells compared to control mice (data not shown). However, $\beta$-CAT-cKO mice had a significant $50 \%$ reduction in the number of iNKT cells (Fig. 2a). $\beta$-CAT-Tg mice, on the other hand, presented a remarkable increase in the number of thymic iNKT cells compared to control mice (Fig. 2b). In light of these data, we conclude that $\beta$-catenin expression is limiting for the generation of thymic iNKT cells.

Analysis of expression of transcription factors PLZF and T-bet in iNKT cell populations from control, $\beta$-CATcKO and $\beta$-CAT-Tg mice showed that the frequency and number of iNKT2 and iNKT17 cells was significantly affected by $\beta$-catenin expression. Specifically iNKT2 and iNKT17 cells, but not iNKT1 cells, accounted for the major decrease in $\beta$-CAT-cKO and the greatest increase in $\beta$-CAT-Tg mice (Fig. 2c-d). We conclude that $\beta$-catenin expression is required for the differentiation and expansion of iNKT2 and iNKT17 cells.

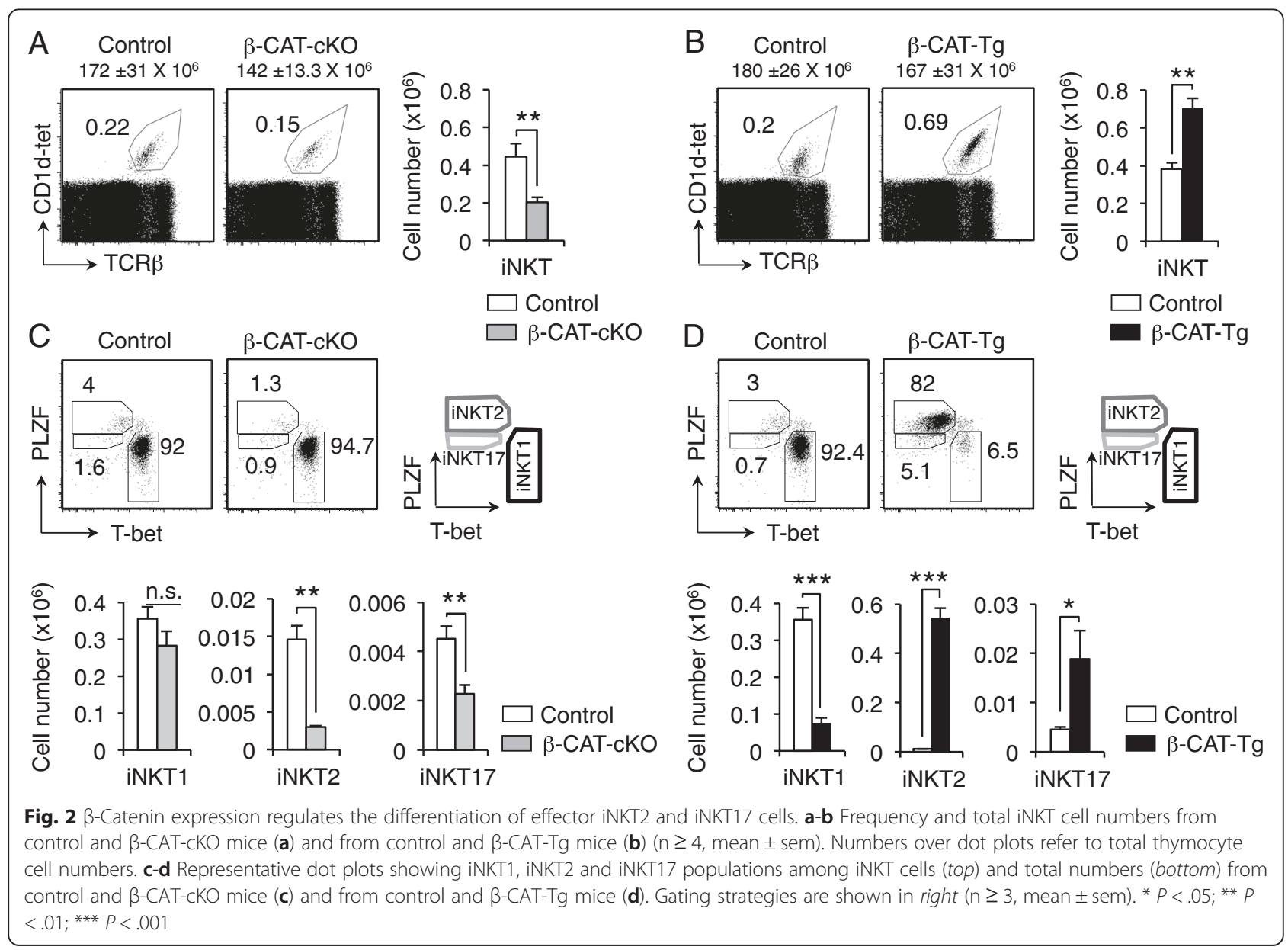


$\beta$-Catenin regulates cytokine production by iNKT cells iNKT cells acquire functional capabilities in the thymus as part of the developmental program. To determine if $\beta$-CAT-Tg iNKT cells were functionally skewed towards a Th2/Th17 phenotype, we cultured $\beta$-CAT-Tg thymocytes in vitro with PMA $(50 \mathrm{ng} / \mathrm{ml})$ and ionomycin $(1 \mu \mathrm{M})$ for $5 \mathrm{~h}$ and then assayed IFN- $\gamma$, IL-4, IL-13 and IL-17 production by thymic iNKT cells. The results showed that the frequency of IFN- $\gamma$-producing iNKT cells from $\beta$-CAT-Tg mice was significantly reduced compared to iNKT cells from control mice (Fig. 3a). Strikingly, a much higher percentage of iNKT cells from $\beta$-CAT-Tg mice expressed IL-4, compared to control iNKT cells, even in unstimulated conditions (Fig. 3a). Likewise, we found enhanced production of IL-13 and also IL-13 + IL-17A+ double-producers by $\beta$-CAT-Tg iNKT cells compared to control iNKT cells (Fig. 3b). We conclude that enforced expression of $\beta$-catenin promotes a higher frequency of Th2- and Th17-like iNKT cells. Importantly, we note that in the absence of stimulation approximately $26 \% \beta$-CAT-Tg thymic iNKT cells produced IL-4 compared to $0.6 \%$ control thymic iNKT cells (Fig. 3a). These data demonstrate that $\beta$-catenin promotes the acquisition of effector functionality in developing iNKT cells in the thymus.

\section{$\beta$-Catenin regulation of iNKT2 and iNKT17 cells is maintained in the periphery}

We then examined the distribution of iNKT cells in the periphery in the $\beta$-catenin mutant mice. We observed that spleen and liver from $\beta$-CAT-cKO mice showed a specific reduction of the iNKT2 and iNKT17 cells in the spleen, and $\beta$-CAT-Tg spleens had reduced iNKT1 and increased iNKT2 and iNKT17 cells (Fig. 4a). Analysis of liver from control, $\beta$-CAT-cKO and $\beta$-CAT-Tg mice showed comparable numbers of iNKT1 cells (Fig. 4a). By contrast, the frequency of cytokine-producing cells was significantly diminished in $\beta$-CAT-cKO mice (Fig. 4a). We conclude that altered iNKT cell development in $\beta$ CAT-cKO and $\beta$-CAT-Tg mice is reflected in the $\beta$ -

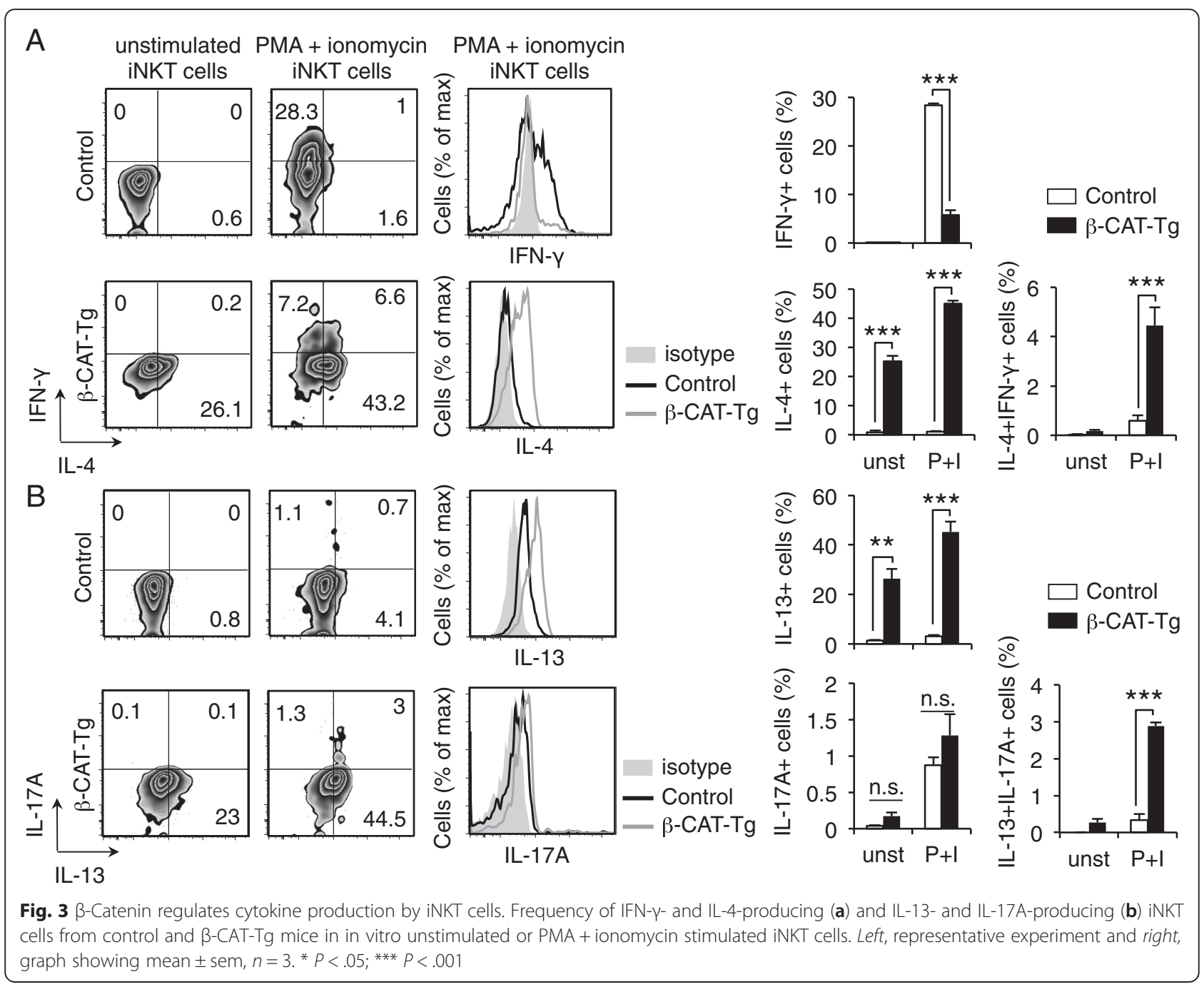




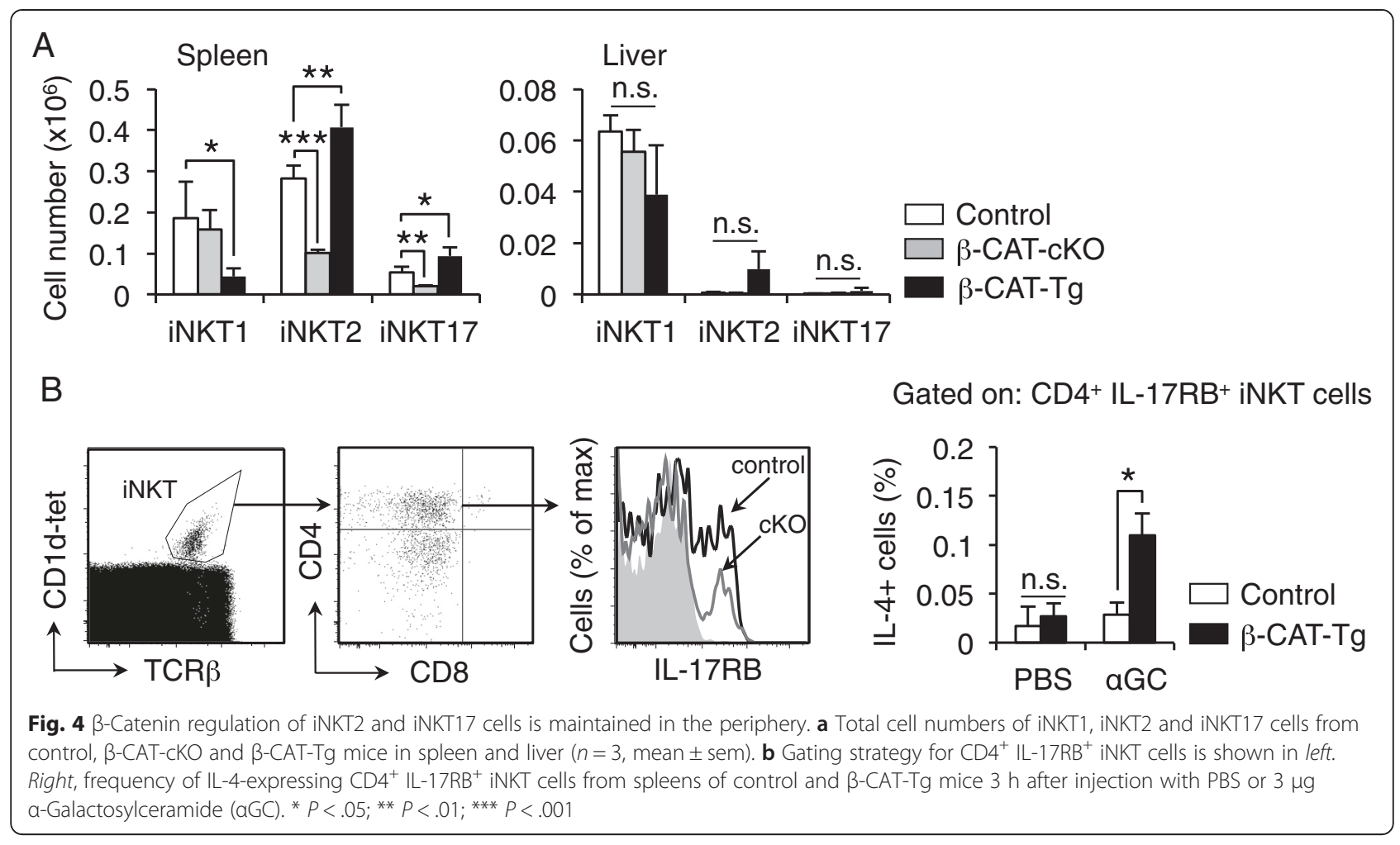

catenin-dependent skewing towards iNKT2 and iNKT17 cells in the distribution of these cells in the periphery.

To further test the efficacy of induced response of Th2-like iNKT2 cells expanded in $\beta$-CAT-Tg mice we tested cytokine production in vivo. To address this we administered $3 \mu \mathrm{g} \alpha$-galactosylceramide $(\alpha G C)$ intraperitoneally to $\beta$-CAT-Tg and control mice and $3 \mathrm{~h}$ postinjection analyzed cytokine production by splenic iNKT cells. $\alpha \mathrm{GC}$ is known to activate iNKT cells in vivo $[1,11$, 35]. Importantly, we found that $\alpha \mathrm{GC}$-stimulated CD4+ IL-17RB+ iNKT cells (gated as in Fig. 4b, left, and mainly iNKT2 cells [29]) in $\beta$-CAT-Tg mice produced high levels of IL-4 (some IL-4+ IFN $\gamma+$ cells were noted but are not included in this analysis) (Fig. 4b, right). Therefore, we conclude that enforced expression of $\beta$ catenin promotes Th2-biased cytokine production by iNKT2 cells in response to stimulation in vivo.

\section{Enhanced generation of $\beta$-catenin-dependent iNKT2 and iNKT17 cells is cell autonomous}

To determine if the effect of enforced $\beta$-catenin expression on iNKT cell generation was cell intrinsic, we generated chimeric mice using either control or $\beta$-CAT-Tg cells as bone marrow donor cells. Analysis of chimeric mice showed that the frequency and numbers of iNKT2 and iNKT17 cells was significantly greater when the donor bone marrow was derived from $\beta$-CAT-Tg mice compared to control mice (Fig. 5a). Furthermore, mixed chimeric mice generated with bone marrow cells derived from wild-type control (CD45.1 + CD45.2+) and from $\beta$ CAT-Tg (CD45.2+) transferred into irradiated wild-type mouse acting as recipient (C57BL/6.SJL, CD45.1+) also showed significantly increased generation of iNKT2 and iNKT17 cells (Fig. 5b). Thus, this data demonstrates that $\beta$-catenin controls differentiation of effector iNKT2 and iNKT17 cells in a cell-intrinsic manner.

\section{Discussion}

In this study we demonstrate that $\beta$-catenin regulates the differentiation of iNKT cells to iNKT2 and iNKT17, but not iNKT1, effector subsets. Conditional deletion of $\beta$-catenin in iNKT cell-precursors impaired differentiation of iNKT2 and iNKT17, but not iNKT1 cells. By contrast, enforced expression of $\beta$-catenin promoted the generation of iNKT2 cells and iNKT17 cells without affecting generation of iNKT1 cells. The effect of enhanced $\beta$-catenin expression in iNKT cell precursors to promote development of iNKT2 and iNKT17 cells was cell-autonomous. Importantly, we show that $\beta$ CAT-Tg iNKT cells acquired the ability to produce enhanced levels of IL-4 and IL-13 during development that augmented lung inflammation in a mouse model for asthma.

Cytokine production by iNKT1, iNKT2 and iNKT17 effector subsets has been shown to be controlled by transcription factors T-bet, GATA3 and ROR $\gamma$ t that also regulate IFN- $\gamma$, IL-4 and IL-17 production by T helper cells [8-10]. However, transcriptional control of 


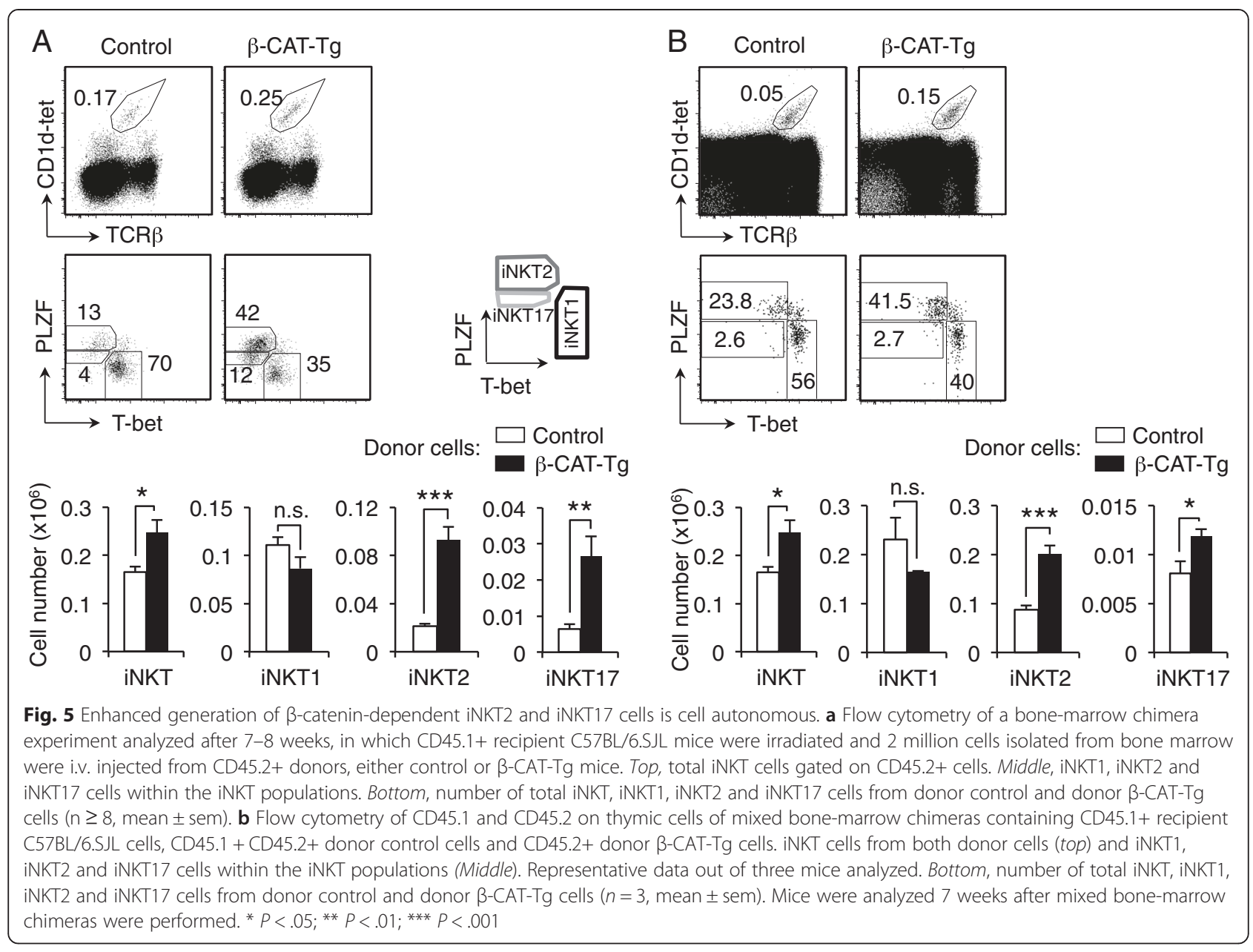

differentiation of iNKT0 cells to iNKT1, iNKT2 and iNKT17 subsets has remained to be defined. This report shows that $\beta$-catenin deficiency resulted in a profound decrease in iNKT2 and iNKT17 subsets of iNKT cells whereas iNKT1 cells developed normally. By contrast, enforced expression of $\beta$-catenin promoted the development of iNKT2 and iNKT17 cells. It was important to note that the majority of iNKT cells in the thymus of C57BL/6 mice were iNKT1 cells and the observation that enforced expression of $\beta$-catenin altered the pattern to iNKT2 and iNKT17 cells suggests that $\beta$-catenin may be a major factor in the distinct pathways that critically direct differentiation of iNKT effector subsets. These observations are congruent with the observation that a cohort of genes (including IL-7R, IL-17RB, Cxcr3, Cxcr6, Ccr4, c-Myc, c-Myb, Bcl11b, Zbtb16, Egr2, Id3, Slamf1 and Slamf6) that have been previously implicated in iNKT cell development were up-regulated in mice with enforced expression of $\beta$-catenin and diminished in expression in cells with deletion of $\beta$-catenin. Unlike effector CD4 T cells, iNKT cells acquire functional capabilities during the differentiation and maturation process. It was interesting to note that $\beta$-CAT-Tg iNKT cells developed the capacity to produce enhanced levels of IL-4, which was reminiscent of the report that TCF1 and $\beta$-catenin promote expression of GATA3 and IL-4 in T helper 2 cells [36]. Thus, we posit that $\beta$-catenin-dependent gene expression regulates the differentiation and functional maturation of iNKT2 and iNKT17 cells.

Finally, our results obtained from the allergic lung inflammation mouse model may be of some clinical importance. Although the precise role for the different human iNKT subsets in the lung and peripheral blood has yet to be clearly defined, correlative studies suggest that iNKT cells are involved in exacerbations of asthma [37, 38]. Furthermore, IL-25 has been reported to be increased in asthmatic humans [39]. Patients with high IL-25- and IL-17RB-containing airway epithelial cells present a more prominent Th2 reaction and respond better to inhaled corticosteroids treatment [40]. We hypothesize that iNKT2 cells may also be increased in IL-25-high asthmatic patients facilitating the generation of "Th2-high" asthma, as 
opposed to a severe, neutrophil-mediated, steroidinsensitive disease. Modula ting the numbers of iNKT cells may be beneficial in reducing asthma symptoms and exacerbations. Targeting novel pathways or molecules involved in iNKT differentiation, such as $\beta$-catenin as we have described here, could be a new approach. In conclusion, this study shows that $\beta$-catenin expression regulates the differentiation of iNKT cells that augment lung inflammation associated with asthma and provides insights that may be of use in designing regimens to limit at least one type of lung inflammation.

\section{Conclusions}

In conclusion data presented in this paper indicate that $\beta$-catenin regulates the differentiation of iNKT cells to iNKT2 and iNKT17, but not iNKT1, effector subsets. We propose that iNKT2 and iNKT17 cells promote asthma and posit that modulating the development and function of these cells provides novel means to control of lung inflammation.

\section{Abbreviations}

iNKT: Invariant Natural Killer T; DP: double positive thymocytes; TCR: T-cell receptor; iCD8: innate-like CD8; IL: Interleukin; AHR: Airway

hyperresponsiveness; IFN- $\gamma$ : Interferon gamma; aGC: a-galactosylceramide.

\section{Competing interests}

The authors declare that they have no competing interests.

\section{Authors' contributions}

JMS, RB-B and AS designed the experiments; RB-B, AS and DAS performed experiments; FCS, KP, Y-HK, C-HC and H-HX contributed chimera experiments; JXF and NMH contributed to the lung inflammation experiments. All authors read and approved the final manuscript.

\section{Authors' information}

Not applicable.

\section{Acknowledgements}

We thank NIA animal facility and genotyping facility for animal husbandry and genotyping, Juliet Meltsner, Kamalvishnu Gottimukkala, Wandi Zhu and Lynette Naler for technical help, Ranjan Sen and Wandi Zhu for insightful comments on the manuscript and the Tetramer Facility of the US National Institutes of Health for providing PE- and APC-conjugated mouse CD1d tetramers loaded with glycolipid PBS-57. This research was supported by the Intramural Research Program of the National Institute on Aging at the National Institutes of Health. Authors declare no conflict of interest

\section{Author details \\ ${ }^{1}$ Immune Cells and Inflammation Section, National Institute on Aging, National Institutes of Health, Baltimore, MD 21224, USA. ${ }^{2}$ Department of Microbiology, Interdisciplinary Immunology Graduate Program, University of lowa, lowa City, IA 52242, USA. ${ }^{3}$ Department of Microbiology and Immunology, The University of Michigan Medical School, Ann Arbor, MI 48109, USA. ${ }^{4}$ Department of Anesthesiology and Critical Care Medicine, The Johns Hopkins University School of Medicine, Baltimore, MD 21287, USA. ${ }^{5}$ Department of Medicine, The Johns Hopkins University School of Medicine, Baltimore, MD 21287, USA. ${ }^{6}$ National Institute on Aging, NIH, Baltimore, MD 21224, USA. ${ }^{7}$ Present addresses: Center for Translational Research, The Feinstein Institute for Medical Research, 350 Community Dr., Manhasset, NY 11030, USA. ${ }^{8}$ Present addresses: Center for Immunology and Microbial Disease, Albany Medical College, Albany, NY 12208, USA.}

Received: 15 July 2015 Accepted: 22 September 2015

Published online: 19 October 2015

\section{References}

1. Bendelac A, Savage PB, Teyton L. The biology of NKT cells. Annu Rev Immunol. 2007;25:297-336.

2. Matsuda JL, Mallevaey T, Scott-Browne J, Gapin L. CD1d-restricted iNKT cells, the 'Swiss-Army knife' of the immune system. Curr Opin Immunol. 2008;20(3):358-68.

3. Brennan PJ, Brigl M, Brenner MB. Invariant natural killer T cells: an innate activation scheme linked to diverse effector functions. Nat Rev Immunol. 2013;13(2):101-17.

4. Van Kaer L, Parekh W, Wu L. Invariant natural killer T cells as sensors and managers of inflammation. Trends Immunol. 2013;34(2):50-8.

5. Bendelac A. Positive selection of mouse NK1+ T cells by CD1-expressing cortical thymocytes. J Exp Med. 1995;182(6):2091-6.

6. Godfrey DI, Stankovic S, Baxter AG. Raising the NKT cell family. Nat Immunol. 2010;11(3):197-206.

7. Hu T, Gimferrer I, Alberola-lla J. Control of early stages in invariant natural killer T-cell development. Immunology. 2011;134(1):1-7.

8. Lee YJ, Holzapfel KL, Zhu J, Jameson SC, Hogquist KA. Steady-state production of IL-4 modulates immunity in mouse strains and is determined by lineage diversity of iNKT cells. Nat Immunol. 2013;14(11):1146-54

9. Cohen NR, Brennan PJ, Shay T, Watts GF, Brigl M, Kang J, et al. Shared and distinct transcriptional programs underlie the hybrid nature of iNKT cells. Nat Immunol. 2013;14(1):90-9.

10. Constantinides MG, Bendelac A. Transcriptional regulation of the NKT cell lineage. Curr Opin Immunol. 2013;25(2):161-7.

11. Gumperz JE, Miyake S, Yamamura T, Brenner MB. Functionally distinct subsets of CD1d-restricted natural killer T cells revealed by CD1d tetramer staining. J Exp Med. 2002;195(5):625-36.

12. Akbari $\mathrm{O}$, Stock $\mathrm{P}$, Meyer $\mathrm{E}$, Kronenberg M, Sidobre $\mathrm{S}$, Nakayama T, et al. Essential role of NKT cells producing IL-4 and IL-13 in the development of allergen-induced airway hyperreactivity. Nat Med. 2003;9(5):582-8.

13. Ikegami $Y$, Yokoyama A, Haruta Y, Hiyama K, Kohno N. Circulating natural killer T cells in patients with asthma. J Asthma. 2004;41(8):877-82.

14. Koh Yl, Shim JU, Wi JO, Han ER, Jin NC, Oh SH, et al. Inverse association of peripheral blood CD4(+) invariant natural killer T cells with atopy in human asthma. Hum Immunol. 2010;71(2):186-91.

15. Magnan A, Mely L, Prato S, Vervloet D, Romagne F, Camilla C, et al. Relationships between natural T cells, atopy, IgE levels, and IL-4 production. Allergy. 2000;55(3):286-90.

16. Shim JU, Koh Yl. Increased Th2-like Invariant Natural Killer T cells in Peripheral Blood From Patients With Asthma. Allergy Asthma Immunol Res. 2014;6(5):444-8.

17. Hamzaoui A, Cheik Rouhou S, Grairi H, Abid H, Ammar J, Chelbi H, et al. NKT cells in the induced sputum of severe asthmatics. Mediators Inflamm. 2006;2006(2):71214.

18. Gounari F, Aifantis I, Khazaie K, Hoeflinger S, Harada N, Taketo MM, et al. Somatic activation of beta-catenin bypasses pre-TCR signaling and TCR selection in thymocyte development. Nat Immunol. 2001;2(9):863-9.

19. Xu Y, Banerjee D, Huelsken J, Birchmeier W, Sen JM. Deletion of betacatenin impairs T cell development. Nat Immunol. 2003;4(12):1177-82.

20. Gounari F, Chang R, Cowan J, Guo Z, Dose M, Gounaris E, et al. Loss of adenomatous polyposis coli gene function disrupts thymic development. Nat Immunol. 2005:6(8):800-9.

21. Staal FJ, Luis TC, Tiemessen MM. WNT signalling in the immune system: WNT is spreading its wings. Nat Rev Immunol. 2008;8(8):581-93.

22. Staal FJ, Sen JM. The canonical Wnt signaling pathway plays an important role in Iymphopoiesis and hematopoiesis. Eur J Immunol. 2008:38(7):1788-94.

23. Yu Q, Sharma A, Sen JM. TCF1 and beta-catenin regulate T cell development and function. Immunol Res. 2010;47(1-3):45-55.

24. Prince AL, Yin CC, Enos ME, Felices M, Berg $L$. The Tec kinases Itk and Rlk regulate conventional versus innate T-cell development. Immunol Rev. 2009;228(1):115-31.

25. Stritesky GL, Jameson SC, Hogquist KA. Selection of self-reactive T cells in the thymus. Annu Rev Immunol. 2012;30:95-114

26. Sharma A, Chen Q, Nguyen T, Yu Q, Sen JM. T cell factor-1 and beta-catenin control the development of memory-like CD8 thymocytes. J Immunol. 2012;188(8):3859-68.

27. Mulroy $T, X u Y$, Sen JM. beta-Catenin expression enhances generation of mature thymocytes. Int Immunol. 2003;15(12):1485-94.

28. Brault V, Moore R, Kutsch S, Ishibashi M, Rowitch DH, McMahon AP, et al. Inactivation of the beta-catenin gene by Wnt1-Cre-mediated deletion results in dramatic brain malformation and failure of craniofacial development. Development. 2001;128(8):1253-64. 
29. Terashima A, Watarai $H$, Inoue $S$, Sekine $E$, Nakagawa $R$, Hase $K$, et al. A novel subset of mouse NKT cells bearing the IL-17 receptor B responds to IL-25 and contributes to airway hyperreactivity. J Exp Med. 2008;205(12):2727-33.

30. Ballantyne SJ, Barlow JL, Jolin HE, Nath P, Williams AS, Chung KF, et al. Blocking IL-25 prevents airway hyperresponsiveness in allergic asthma. J Allergy Clin Immunol. 2007;120(6):1324-31.

31. Fort MM, Cheung J, Yen D, Li J, Zurawski SM, Lo S, et al. IL-25 induces IL-4, $\mathrm{IL}-5$, and IL-13 and Th2-associated pathologies in vivo. Immunity. 2001;15(6):985-95

32. Suzukawa M, Morita H, Nambu A, Arae K, Shimura E, Shibui A, et al. Epithelial cell-derived IL-25, but not Th17 cell-derived IL-17 or IL-17 F, is crucial for murine asthma. J Immunol. 2012;189(7):3641-52.

33. DeKruyff RH, Yu S, Kim HY, Umetsu DT. Innate immunity in the lung regulates the development of asthma. Immunol Rev. 2014;260(1):235-48.

34. Stock $\mathrm{P}$, Lombardi $\mathrm{V}$, Kohlrautz $\mathrm{V}$, Akbari $\mathrm{O}$. Induction of airway hyperreactivity by IL-25 is dependent on a subset of invariant NKT cells expressing IL-17RB. J Immunol. 2009;182(8):5116-22.

35. Kawano T, Cui J, Koezuka Y, Toura I, Kaneko Y, Motoki K, et al. CD1drestricted and TCR-mediated activation of valpha14 NKT cells by glycosylceramides. Science. 1997;278(5343):1626-9.

36. Yu Q, Sharma A, Oh SY, Moon HG, Hossain MZ, Salay TM, et al. T cell factor 1 initiates the $T$ helper type 2 fate by inducing the transcription factor GATA-3 and repressing interferon-gamma. Nat Immunol. 2009;10(9):992-9.

37. Juno JA, Keynan Y, Fowke KR. Invariant NKT cells: regulation and function during viral infection. PLoS Pathog. 2012;8(8), e1002838.

38. Carpio-Pedroza JC, Vaughan G, del Rio-Navarro BE, del Rio-Chivardi JM Vergara-Castaneda A, Jimenez-Zamudio LA, et al. Participation of CD161(+) and invariant natural killer T cells in pediatric asthma exacerbations. Allergy Asthma Proc. 2013;34(1):84-92.

39. Corrigan CJ, Wang W, Meng Q, Fang C, Eid G, Caballero MR, et al. Allergeninduced expression of IL-25 and IL-25 receptor in atopic asthmatic airways and late-phase cutaneous responses. J Allergy Clin Immunol. 2011;128(1):116-24.

40. Cheng D, Xue Z, Yi L, Shi H, Zhang K, Huo X, et al. Epithelial interleukin-25 is a key mediator in th2-high, corticosteroid-responsive asthma. Am J Respir Crit Care Med. 2014;190(6):639-48

\section{Submit your next manuscript to BioMed Central and take full advantage of:}

- Convenient online submission

- Thorough peer review

- No space constraints or color figure charges

- Immediate publication on acceptance

- Inclusion in PubMed, CAS, Scopus and Google Scholar

- Research which is freely available for redistribution 